\title{
Aloperine induces G2/M phase cell cycle arrest and apoptosis in HCT116 human colon cancer cells
}

\author{
LI ZHANG $^{1 *}$, YANXIN ZHENG ${ }^{1 *}$, HONGZHU DENG ${ }^{1}$, LEI LIANG $^{2}$ and JUAN PENG ${ }^{3}$ \\ ${ }^{1}$ School of Traditional Chinese Medicine, Southern Medical University, Guangzhou, Guangdong 510515; \\ ${ }^{2}$ Department of Pharmacy, Guangzhou General Hospital of Guangzhou Military Command, Guangzhou, Guangdong 510010; \\ ${ }^{3}$ Department of Pharmacy, Hunan Loudi Central Hospital, Loudi, Hunan 417000, P.R. China
}

Received December 3, 2013; Accepted March 20, 2014

DOI: $10.3892 / \mathrm{ijmm} .2014 .1718$

\begin{abstract}
Aloperine (ALO) is a quinolizidine alkaloid extracted from the leaves of Sophora alopecuroides (S. alopecuroides) and possesses anti-inflammatory, anti-allergenic, antitumor, and antiviral effects. In this study, when compared with seven other types of alkaloids extracted from $S$. alopecuroides, ALO treatment produced the most potent effects against HCT116 colon cancer cell types. ALO inhibited proliferation and induced apoptosis in HCT116 cells in a doseand time-dependent manner as detected by MTT, clonogenic survival, and flow cytometric assays. Results of the western blot analysis and qPCR revealed that ALO increased the protein and mRNA of Bax and decreased Bcl-2 via the mitochondrial death pathway. In addition, ALO induced cell cycle arrest at the $\mathrm{G} 2 / \mathrm{M}$ phase with a concomitant increase in p21 and p53 and a decrease in cyclin D1 and B1. ALO also inhibited phosphatidylinositol 3-kinase/Akt and JAK/Stat3. Generally, ALO exerted a significant anti-proliferative effect via apoptotic and cell cycle arrest induction in HCT116 cells. These results suggested that ALO should be investigated further as an agent of chemotherapeutic activity in human colon cancer.
\end{abstract}

\section{Introduction}

Colon cancer is the third most common cancer. A recent decline in the incidence of colon cancer has been largely attributed to an increase in screening for the disease, allowing for the detection and removal of precancerous polyps. However, the mortality rate associated with colon cancer remains high (1). Establishing treatment strategies that reduce unfavorable effects and increase the overall prognosis of colon cancer is challenging. An inverse

Correspondence to: Professor Hongzhu Deng, School of Traditional Chinese Medicine, Southern Medical University, 1023 Shatai Southern Road, Guangzhou, Guangdong 510515, P.R. China

E-mail: 1098631571@qq.com

*Contributed equally

Key words: aloperine, cell cycle, G2/M, apoptosis, HCT116, colon cancer correlation between a high intake of fruits, vegetables, and phytochemicals and a reduced risk of colon cancer has been reported (2). The chemopreventive agents and their constituent phytochemicals derived from plants have been known to interfere with various molecular pathways involved in colon cancer initiation and progression (3). Therefore, there is considerable interest in developing preventive and therapeutic agents for cancer from natural products (4).

Sophora alopecuroides ( $S$. alopecuroides) is a traditional Chinese herb that has been used clinically for acute bacillary dysentery and enteritis. Its main bioactive components are quinolizidine alkaloids, including $>20$ types of alkaloids (5). Most of the components, such as matrine, oxymatrine and sophoridine, possess antitumor activities (6-11).

Aloperine (ALO) (Fig. 1) is a quinolizidine alkaloid that is extracted from S. alopecuroides (12). ALO exerts anti-inflammatory, anti-allergenic, antitumor and antiviral effects (12-15). Of six alkaloids derived from $S$. alopecuroides, ALO was found to exert the most potent cytotoxic activity against several human cancer cells of differing tissue origins (13). However, little is known with regard to the effect of ALO against colon cancer and the mechanisms associated with its activity.

The aim of the present study was to assess the antitumor efficacy of ALO and other alkaloids isolated from $S$. alopecuroides, including matrine, oxymatrine, sophoridine, sophocarpine, oxysophocarpine, lehmannine and cytisine (Fig. 1) against HCT116 colon cancer cells and to investigate the mechanism of ALO resistance of colon cancer cells to provide some basis for the development of ALO as a new drug against colon cancer. The data showed that the series of alkaloids isolated from S.alopecuroides inhibited the growth of HCT116 cells and that ALO possesses the strongest cytotoxic activity against these cells compared with the other alkaloids. In HCT116 human colon cancer cells, ALO also produced long-term clonogenic effects. To identify the molecular mechanisms underlying these responses to ALO, we investigated the effects of ALO on cell cycle progression and apoptosis.

\section{Materials and methods}

Materials. Matrine, oxymatrine, sophoridine and oxysophocarpine were purchased from the National Institutes for Food 
<smiles>[R]N1CCCC2=C[C@@H]3C[C@H](CN4CCCC[C@H]34)[C@@H]2C1</smiles>

Aloperine

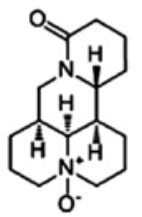

Oxymatrine

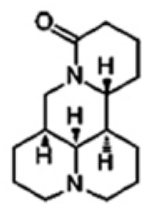

Matrine

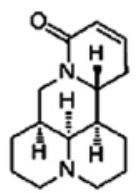

Sophocarpine

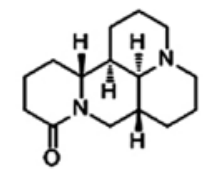

Sophoridin

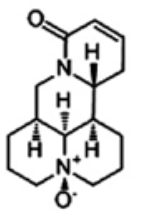

Oxysophocarpine

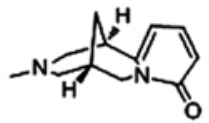

Cytisine

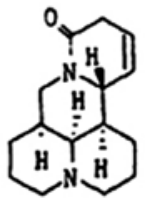

Lehmannine

Figure 1. Chemical structure of the elements.

and Drug Control (Beijing, China). ALO, sophocarpine, lehmannine and cytisine were generously provided by the Ningxia Bauhinia Pharmaceutical Co., Ltd. (Ningxia, China). All of the compounds were dissolved in phosphate-buffered saline (PBS; Boster, Wuhan, China) at a concentration of $20 \mathrm{mM}$ and stored at $4^{\circ} \mathrm{C}$.

Cell culture. HCT116 human colon cancer cells were purchased from the American Type Culture Collection (Manassas, VA, USA). The cells were cultured in RPMI-1640 medium (HyClone, Thermo Fisher Biotechnology, Beijing, China) supplemented with $10 \%$ fetal bovine serum (FBS; Tianhang, Hangzhou, China), $100 \mathrm{U} / \mathrm{ml}$ penicillin and $10 \mathrm{mg} / \mathrm{ml}$ streptomycin (Gibco, Life Technologies Biotechnology, Shanghai, China) and grown in a $37^{\circ} \mathrm{C}$ incubator with $5 \% \mathrm{CO}_{2}$. The medium was replaced at 2-3 day intervals. Subconfluent cells were routinely harvested with $0.05 \%$ trypsin/0.02\% EDTA (Gibco, Life Technologies Biotechnology).

MTT assay. HCT116 cells were seeded at $1 \times 10^{4}$ cells/well in $180 \mu \mathrm{l}$ of complete culture medium in 96-well plates and cultured for $24 \mathrm{~h}$. The medium was then replaced with fresh RPMI-1640 or with fresh RPMI-1640 containing various concentrations of ALO $(0,0.3125,0.625,1.25,2.5$ and $5 \mathrm{mM})$ and the other alkaloids $(0,1.25,2.5,5,7.5$ and $10 \mathrm{mM})$. Each alkaloid concentration was repeated in 5 wells. After incubating for 24,48 or $72 \mathrm{~h}$, MTT $(5 \mathrm{mg} / \mathrm{ml}, 20 \mu \mathrm{l})$ was added to each well and incubated for $4 \mathrm{~h}$. The medium was aspirated from the wells and $150 \mu \mathrm{l}$ of dimethyl sulfoxide (DMSO; Fuyu Chemical, Tianjin, China) was added into each well and agitated for $10 \mathrm{~min}$. The optical density (OD) at $570 \mathrm{~nm}$ was recorded using a microplate reader (Thermo Scientific, Multiskan GO, Waltham, MA, USA). The inhibition rate was calculated using the formula: inhibition rate $(\%)=\left(1-\mathrm{A}_{\text {treated }} / \mathrm{A}_{\text {control }}\right) \times 100 \%$.

Observation of morphologic changes. HCT116 cells were seeded into 6-well plates and treated with various concentrations $(0,0.25,0.5$ and $1 \mathrm{mM})$ of ALO for $24 \mathrm{~h}$. Cell morphology was observed using a fluorescence microscope (Eclipse T1; Nikon, Tokyo, Japan).
Clonogenic survival assay. HCT116 cells were counted and seeded into 6 -well tissue culture plates $\left(3 \times 10^{2}\right.$ cells/well) in RPMI-1640 supplemented with 10\% FBS. After the cells adhered, they were treated with different doses $(0,0.1,0.2$ and $0.3 \mathrm{mM}$ ) of ALO for 7 days. The cells were then fixed with $4 \%$ paraformaldehyde (PFA) and stained with $0.1 \%$ crystal violet (Beyotime Institute of Biotechnology, Jiangsu, China) (16). Representative images were captured using a digital camera (Olympus, Tokyo, Japan).

Flow cytometric (FACS) analysis. HCT116 cells were seeded into 6-well plates at a density of $1 \times 10^{6}$ cells/well and were treated with a series of ALO concentrations $(0,0.25$ and $0.5 \mathrm{mM}$ ) for $24 \mathrm{~h}$. The cells were collected, washed with PBS and fixed in $70 \%$ ethanol, and then stored at $4^{\circ} \mathrm{C}$ overnight. The cells were washed with PBS again and stained with $5 \mu \mathrm{g} / \mathrm{ml}$ RNase and $20 \mu \mathrm{g} / \mathrm{ml}$ of propidium iodide (PI) (Beyotime Institute of Biotechnology) in the dark at $37^{\circ} \mathrm{C}$ for $30 \mathrm{~min}$ and analyzed using a flow cytometer (FACScan; BD Biosciences, Franklin Lakes, NJ, USA). The cellular DNA contents were identified for detection of the cell cycle distribution. At least 10,000 events were counted for each sample.

Apoptosis was measured using the Annexin V-FITC/ PI Apoptosis Detection kit (Invitrogen, Carlsbad, CA, USA) according to the manufacturer's instructions. HCT116 cells $\left(1 \times 10^{6}\right.$ cells/well) were treated with a series of ALO concentrations $(0,0.25,0.5$ and $1 \mathrm{mM})$ for $24 \mathrm{~h}$. The cells were then collected by centrifugation and washed with PBS. The cells were centrifuged and resuspended in $100 \mu \mathrm{l}$ of binding buffer to which $5 \mu \mathrm{l}$ of Annexin $\mathrm{V}$ and $1 \mu \mathrm{l}$ of PI were added. The mixture was incubated at room temperature in the dark for $15 \mathrm{~min}$. The cells were analyzed using a flow cytometer. For each measurement, at least 10,000 cells were counted.

Western blot analysis. The cells were incubated with a series of ALO concentrations $(0,0.25,0.5$ and $1 \mathrm{mM})$ for $24 \mathrm{~h}$ and harvested. The cells were lysed in RIPA lysis buffer (Beyotime Institute of Biotechnology) on ice for $30 \mathrm{~min}$. The proteins contained in the lysates were quantified using the BCA Protein assay kit (Beyotime Institute of Biotechnology). Total protein $(30 \mu \mathrm{g})$ was subjected to $10 \%$ SDS polyacrylamide 
gel electrophoresis (Beyotime Institute of Biotechnology) and transferred onto PVDF membranes. The membranes were blocked with $5 \%$ non-fat milk at room temperature for $2 \mathrm{~h}$ with rocking and then incubated with specific primary antibodies against p53, Bax, Bcl-2, cyclin D1, p21, cyclin B1, JAK1, Stat3, p101 (PI3KC3), Akt and $\beta$-actin (Cell Signaling Technology, Beverly, MA, USA) overnight at $4^{\circ} \mathrm{C}$. After washing the membranes with TBS (PBS with $0.05 \%$ Tween-20) three times for $15 \mathrm{~min}$ each, the membranes were incubated with secondary antibodies at room temperature for $2 \mathrm{~h}$. After washing three times in TBS for $15 \mathrm{~min}$, the specific protein bands were detected using the Kodak Image Station (Eastman Kodak, Rochester, NY, USA). The data were normalized to $\beta$-actin for analyses and plotting.

Quantitative RT-PCR ( $R R T-P C R)$ assays. Total RNA was isolated from the cells using a TRIzol RNA isolation kit (Invitrogen). The cDNAs were synthesized using the PrimeScript $^{\mathrm{TM}}$ II first-strand cDNA synthesis kit (Takara, Dalian, China) according to the manufacturer's instructions. The qRT-PCR reactions were performed in triplicate on an ABI PRISM 7300 (Applied Biosystems, Foster City, CA, USA) using the C1000 thermal cycler PCR (Bio-Rad, Hercules, CA, USA). Primer sequences used in this study are provided in Table I. The PCR conditions included an initial denaturation step at $93^{\circ} \mathrm{C}$ for $2 \mathrm{~min}$, followed by 40 cycles of a denaturation step at $93^{\circ} \mathrm{C}$ for $15 \mathrm{sec}$ and an annealing/extension step at $55^{\circ} \mathrm{C}$ for $25 \mathrm{sec}$ and $72^{\circ} \mathrm{C}$ for $25 \mathrm{sec}$. The relative expression values of the different genes were calculated using the $2^{-\Delta \Delta \mathrm{Ct}}$ method.

Statistical analysis. Data are presented as the mean \pm SD of at least three independent experiments. The Student's t-test and ANOVA were used to identify statistically significant differences with SPSS 13.0 software. Differences were considered significant when $\mathrm{P}<0.05$. The $\mathrm{IC}_{50}$ values were calculated with SPSS 13.0 software using Probit analysis.

\section{Results}

ALO inhibits the proliferation and clonogenic survival of HCT116 colon cancer cells. To assess the activity of ALO as a therapeutic agent against colon cancer, we examined the effects of ALO and other alkaloids on the proliferation of HCT116 colon cancer cells. Exponentially increasing HCT116 cells were treated with various concentrations of ALO and other alkaloids for 24, 48 and $72 \mathrm{~h}$. These drugs notably decreased the viability of HCT116 cells as detected by the MTT assay (Fig. 2A). Compared with the other alkaloids, ALO exhibited the most potent cytotoxic properties. Oxymatrine and oxysophocarpine produced the lowest cytotoxic properties of all the alkaloids tested, while sophoridine, sophocarpine, lehmannine, cytosine and matrine exhibited similar functions. ALO decreased the percentage of adherent HCT116 cells in a concentration- and time-dependent manner (Fig. 2B). Additionally, the ALO treatment induced obvious morphological changes. Compared with the control group, the treated cells were more round and exhibited more floating dead cells (Fig. 2C). To investigate the anticancer effect of ALO in HCT116 human colon cancer cells, the colony
Table I. Primer sequences.

\begin{tabular}{|c|c|c|}
\hline Genes & Primer sequences & $\begin{array}{l}\text { Amplied } \\
\text { fragment } \\
\text { length (bp) }\end{array}$ \\
\hline Bax & $\begin{array}{l}\text { F: 5'-GCTGGACATTGGACTTCCTC-3' } \\
\text { R: 5'-CTCAGCCCATCTTCTTCCAG-3' }\end{array}$ & 129 \\
\hline Bcl-2 & $\begin{array}{l}\text { F: 5'-ATGTGTGTGGAGAGCGTCAA-3' } \\
\text { R: 5'-ACAGTTCCACAAAGGCATCC-3' }\end{array}$ & 136 \\
\hline Tp53 & $\begin{array}{l}\text { F: 5'-CCAGCCAAAGAAGAAACCAC-3' } \\
\text { R: 5'-CCTCATTCAGCTCTCGGAAC-3' }\end{array}$ & 92 \\
\hline JAK1 & $\begin{array}{l}\text { F: 5'-TGCCATGATGAAGAAGATGC-3' } \\
\text { R: 5'-GACACGCTGCTGTCACAAAT-3' }\end{array}$ & 186 \\
\hline Stat3 & $\begin{array}{l}\text { F: 5'-AGTATAGCCGCTTCCTGCAA-3' } \\
\text { R: 5'-GCAATCTCCATTGGCTTCTC-3' }\end{array}$ & 106 \\
\hline AKT1 & $\begin{array}{l}\text { F: 5'-AGAAGCAGGAGGAGGAGGAG-3' } \\
\text { R: 5'-CCCAGCAGCTTCAGGTACTC-3' }\end{array}$ & 139 \\
\hline PI3K & $\begin{array}{l}\text { F: 5'-AAGCAGTGCCTGTAGGAGGA-3' } \\
\text { R: 5'-TGTCGATGAGCTTTGGTGAG-3' }\end{array}$ & 199 \\
\hline Cyclin B1 & $\begin{array}{l}\text { F: 5'-TTGATACTGCCTCTCCAAGCC-3' } \\
\text { R: 5'-AGCTCCATCTTCTGCATCCAC-3' }\end{array}$ & 122 \\
\hline H-P21 & $\begin{array}{l}\text { F: 5'-TTGTACCCTTGTGCCTCGCT-3' } \\
\text { R: 5'-AATCTGTCATGCTGGTCTGCC-3' }\end{array}$ & 101 \\
\hline Cyclin D1 & $\begin{array}{l}\text { F: 5'-ACCTGAGGAGCCCCAACAAC-3' } \\
\text { R: 5'-GCTTCGATCTGCTCCTGGC-3' }\end{array}$ & 112 \\
\hline H- $\beta$-actin & $\begin{array}{l}\text { F: 5'-GCATGGGTCAGAAGGATTCCT-3' } \\
\text { R: 5'-TCGTCCCAGTTGGTGACGAT-3' }\end{array}$ & 106 \\
\hline
\end{tabular}

F, forward primer; R, reverse primer; PI3K, phosphatidylinositol 3-kinase.

formation assay was used. After long-term ALO treatment, clonogenicity was markedly reduced in the ALO-treated cells compared with the control group (Fig. 2D). The clonogenic number of HCT116 cells decreased after the treatment with different ALO concentrations, which is consistent with the results of the morphological changes and MTT assay. Thus, the results indicated that ALO induces anti-proliferative activity in HCT116 colon cancer cells.

ALO inhibits cell cycle progression in HCT116 cells. Cell cycle arrest and apoptosis induction are two major causes of cell proliferative inhibition. To establish whether ALO influences cell cycle progression, we determined the distribution of ALO-treated HCT116 cells in various cell cycle stages using flow cytometry. ALO treatment ( $24 \mathrm{~h}$ ) effectively mediated G2 cell cycle arrest in HCT116 cells (Fig. 3A). As expected, the number of G2 and S phase cells was increased after $24 \mathrm{~h} \mathrm{ALO}$ treatment (Fig. 3B). The cell cycle analysis of the HCT116 cells treated with different concentrations of ALO revealed a higher number of cells in the G2/M and S phases, whereas the number of cells in G0/G1 phase decreased compared with the untreated cells. This result indicated that inhibition of the cell cycle progression is an upstream event leading to apoptosis. To resolve ALO-mediated G2 cell cycle arrest, the effects of 

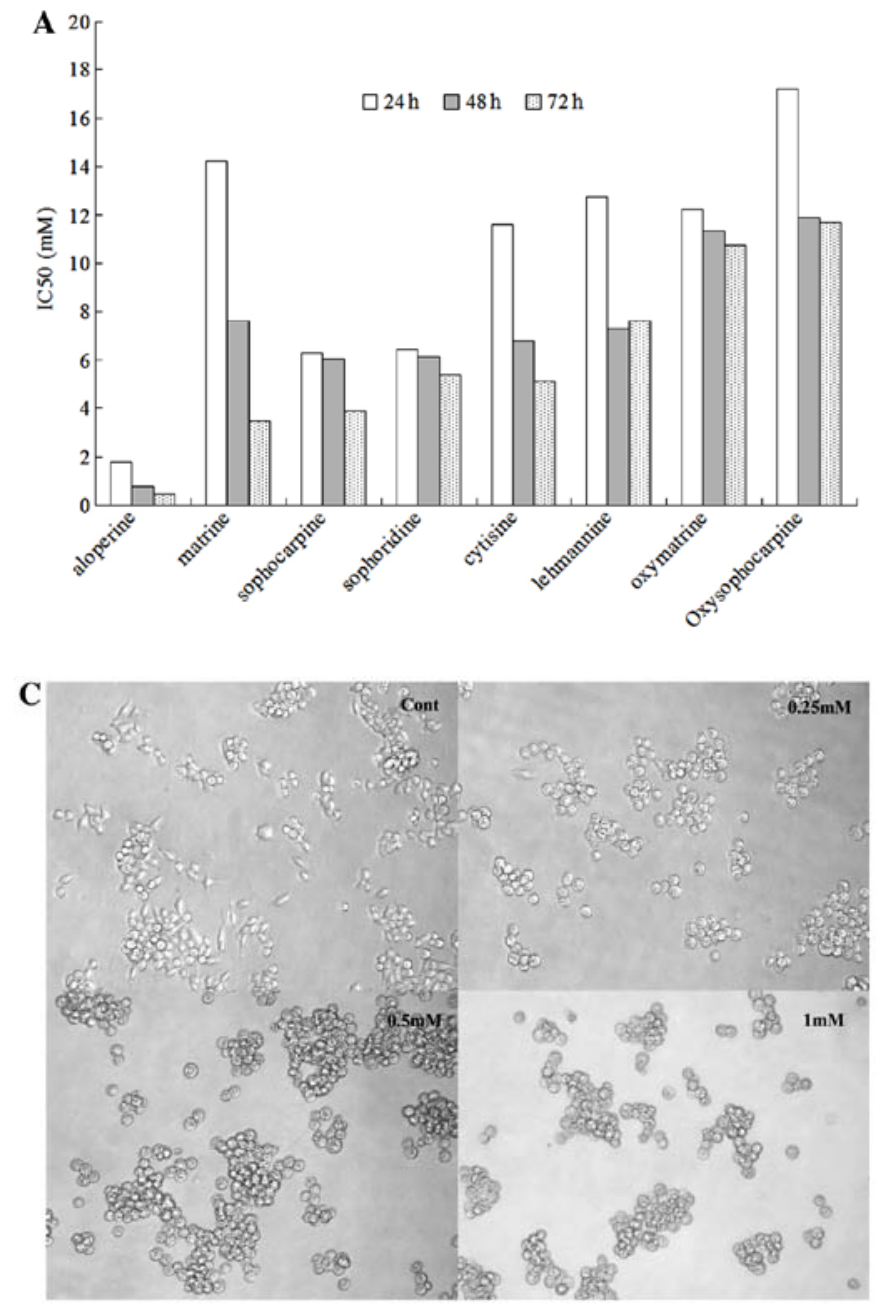

ALO on the expression of cell cycle regulatory proteins (p53, p21, cyclin B1 and cyclin D1) were examined by western blot analysis (Fig. 3C). Results of the western blot analysis indicated that the levels of p53 and p21 increased after exposure to ALO for $24 \mathrm{~h}$. By contrast, the levels of cyclin D1 and B1 were significantly reduced at $24 \mathrm{~h}$. The PCR analysis results concurred with those of the western blot analysis (Fig. 3D). These results suggested that ALO deregulates cell cycle progression and induces apoptosis.

ALO induces apoptosis in HCT116 cancer cells. Apoptotic assays were performed to determine whether ALO induced apoptosis in HCT116 cells. The cells were stained with Annexin V and PI and analyzed by flow cytometry. The early apoptotic death rate in HCT116 cells treated with increasing concentrations of ALO was not significantly different from the control group (Fig. 4A). A few late apoptotic cells were observed in the control group. However, in the ALO-treated group, large amounts of apoptotic cells were found. The rate of late apoptotic cells in the control group was $<5.0 \%$, while the treatment with various concentrations of ALO for $24 \mathrm{~h}$ significantly increased the incidence of late apoptotic cells, and the rate of late apoptosis reached $4.99 \pm 1.37,14.93 \pm 2.20$ and $17.40 \pm 2.93 \%(\mathrm{P}<0.01)$ with ALO concentrations of 0.25 , 0.5 and $1 \mathrm{mM}$, respectively (Fig. 4B). The rate of late apoptosis was increased in a concentration-dependent manner.
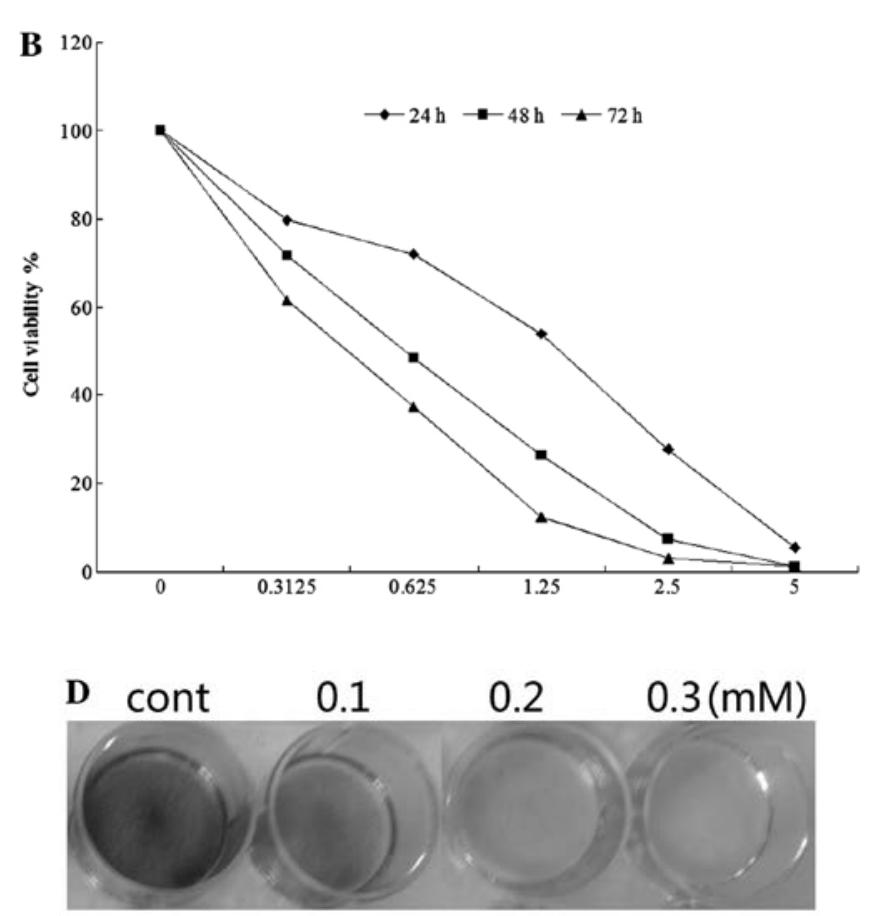

Figure 2. Anti-proliferative effects of aloperine (ALO) on HCT116 colon cancer cells. (A) Growth inhibitory activities of eight types of alkaloids in HCT116 cells following the comparison of $\mathrm{IC}_{50}$ values. Data are shown as means \pm standard deviation (SD) of three independent experiments. (B) Cell anti-proliferation assay. HCT116 cells were treated with different doses of ALO for 24, 48 and $72 \mathrm{~h}$ and the cell viability was tested by MTT assay. (C) The morphological changes of HCT116 cells following ALO treatment. Original magnification, x200. (D) Dose-dependent clonogenic survival assay. Representative images of colony formation assay were captured.

ALO induces cell death by activating the mitochondrial apoptotic pathway. The Bcl-2 family of proteins plays a crucial role in regulating the mitochondria-dependent pathway of apoptosis. Therefore, we examined whether Bcl-2 and Bax regulation was involved in ALO-induced apoptosis using RT-PCR and western blotting to detect their expression. Bcl-2 was downregulated, and Bax expression was upregulated following ALO treatment (Fig. 4C and D). Therefore, the results indicated that ALO induced apoptosis, which was accompanied by the dose-dependent upregulation of Bax and downregulation of $\mathrm{Bcl}-2(\mathrm{P}<0.05)$.

Effects of ALO on JAK/Stat 3 and phosphatidylinositol 3-kinase (PI3K)/Akt signaling. PI3K/Akt and JAK/Stat3 signaling pathways regulate cell proliferation, survival, and apoptosis (17-19). Therefore, the effects of ALO on the modification of apoptosis-related proteins and mRNA were examined. The HCT116 cells treated with ALO for $24 \mathrm{~h}$ demonstrated a dose-dependent decrease in mRNA and protein in Stat 3 and PI3KC3. By contrast, modest dose-dependent decreases were detected in JAK1 and Akt (Fig. 5).

\section{Discussion}

The identification of effective anticancer drugs from plant-derived natural products plays an important role in 
A
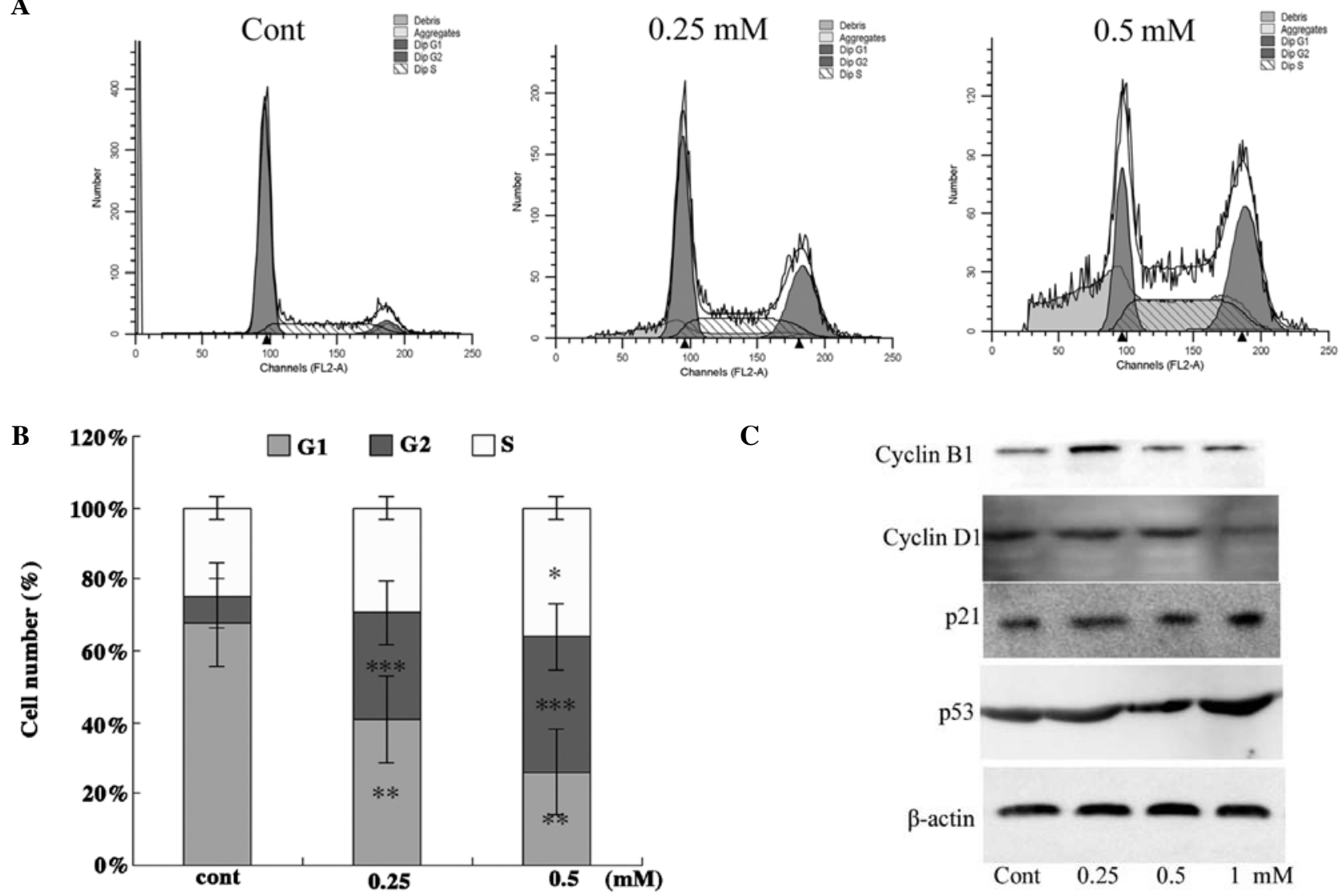

C
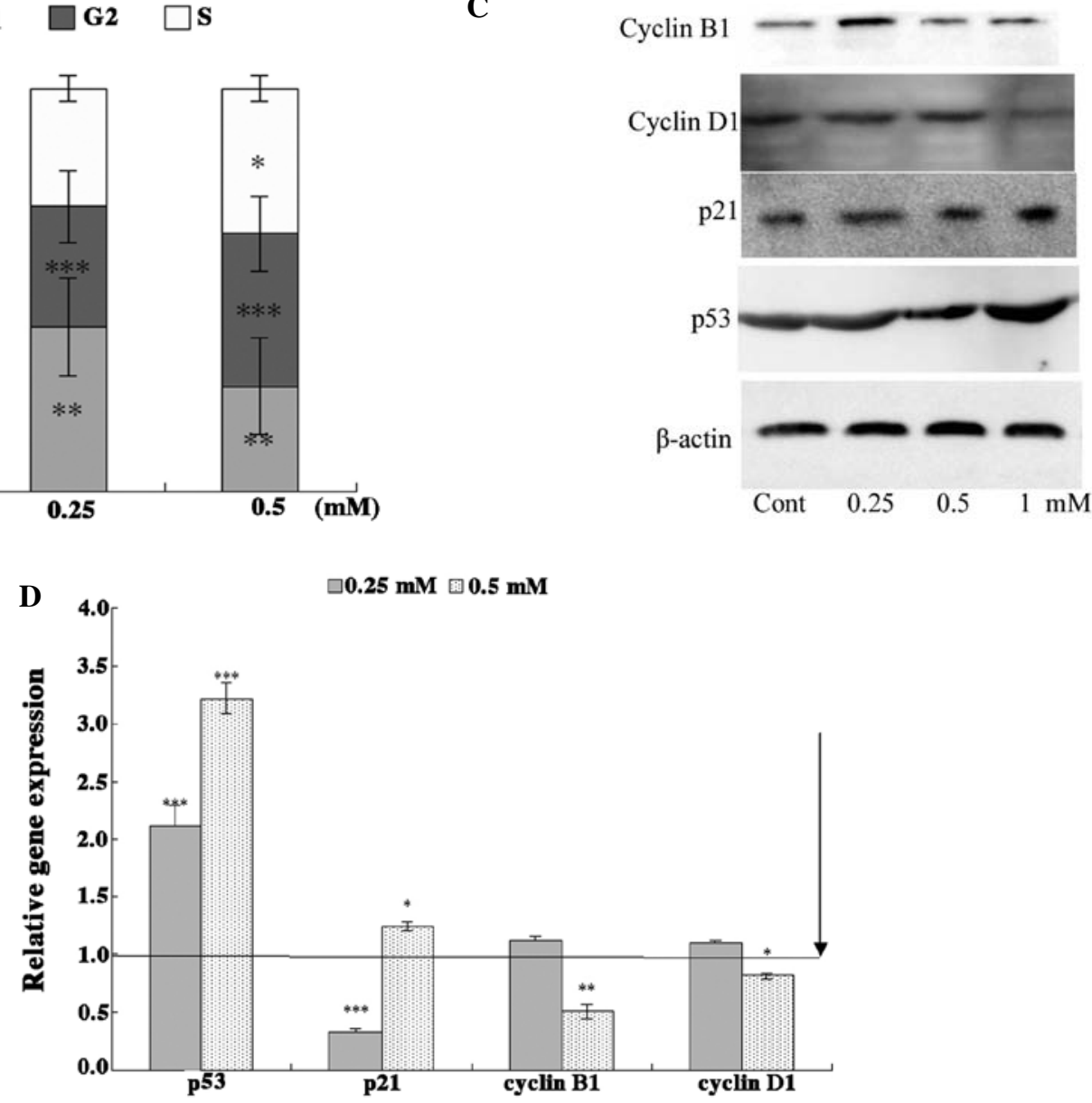

Figure 3. Effect of aloperine (ALO) on cell cycle progression. (A) FACS analysis. Results shown are representative of three independent experiments. (B) Analysis of the cell cycle with different concentrations of aloperine (ALO) in HCT116 cells. The result shows that the number of G2 and S phase cells was increased, whereas the number of cells in G0/G1 phase decreased compared to the untreated cells. Data are shown as the mean \pm standard deviation (SD) of three independent experiments. ${ }^{*} \mathrm{P}<0.05,{ }^{* *} \mathrm{P}<0.01$ and ${ }^{* * *} \mathrm{P}<0.001$. (C) Expression of cell cycle-related proteins. The results shown are representative of three independent experiments. (D) Relative expression of selected cell cycle arrest-related genes examined by qPCR array. Representative up- and downregulated genes are shown. Data are expressed as mean $\pm \mathrm{SD}$, relative gene expression was normalized using $\beta$-actin as a control $\left({ }^{(*} \mathrm{P}<0.05,{ }^{* *} \mathrm{P}<0.01\right.$ and $\left.{ }^{* * *} \mathrm{P}<0.001\right)$.

cancer chemotherapy. Alkaloids constitute a rich resource of compounds for identifying drugs. Most of the alkaloids identified in $S$. alopecuroides are quinolizidine alkaloids. Previous studies have confirmed that some quinolizidine alkaloids, including matrine, oxymatrine and sophoridine, exert a marked anticancer effect and are believed to be beneficial for cancer chemoprevention (6-11). ALO demonstrated a more effective inhibition of several cancer cell lines than matrine, oxymatrine and sophoridine (13). However, there are no reports that address the effect of ALO on colon cancer cells or whether ALO is more effective than other quinolizidine alkaloids. The mechanisms underlying the antitumor activity of ALO have not been studied in detail. In this study, we investigated the antitumor activities of the alkaloids in vitro. Compared to seven other alkaloids, ALO produced the most potent time- and dose-dependent inhibition of proliferation of HCT116 colon cancer cells. ALO effectively inhibited the long-term clonogenic survival of HCT116 cells by inhibiting 
A
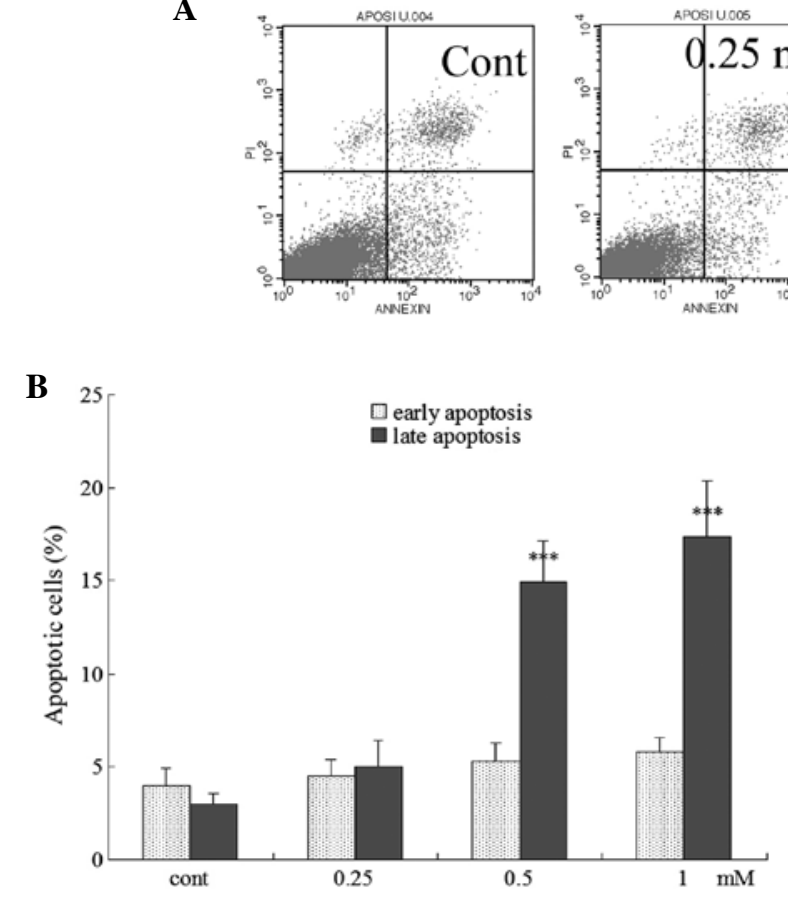
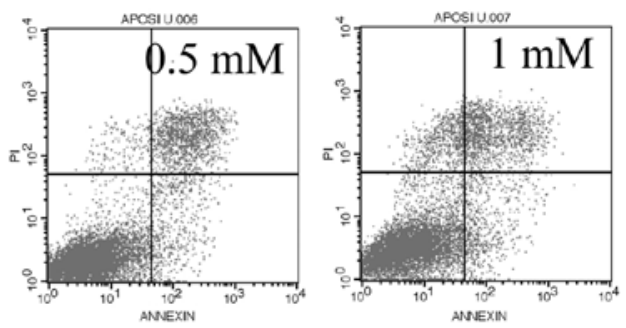

C

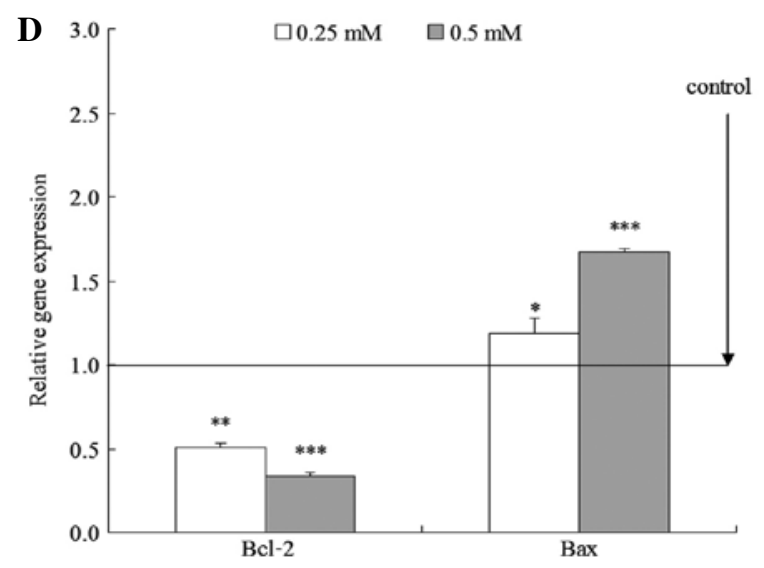

Figure 4. Aloperine (ALO) induces apoptosis in HCT116 cells. (A) FACS analysis. Results shown are representative of three independent experiments. (B) Representative histograms for apoptotic rate in HCT116 cells $\left.{ }^{(* * * *} \mathrm{P}<0.001\right)$. (C) Expression of Bcl-2 and Bax proteins. The results shown are representative of three independent experiments. (D) Relative expression of Bcl-2 and Bax genes examined by qPCR array. Representative up- and downregulated genes are shown. Data are expressed as the mean \pm standard deviation (SD), and the relative gene expression was normalized using $\beta$-actin as a control. ("P<0.05, ${ }^{* *} \mathrm{P}<0.01$ and $\left.{ }^{* * *} \mathrm{P}<0.001\right)$.

A

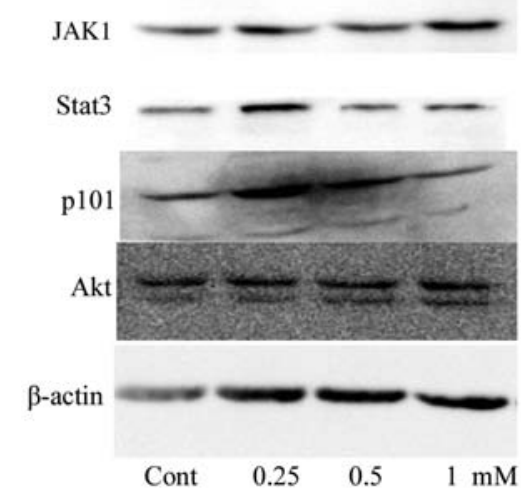

$\mathbf{B}$

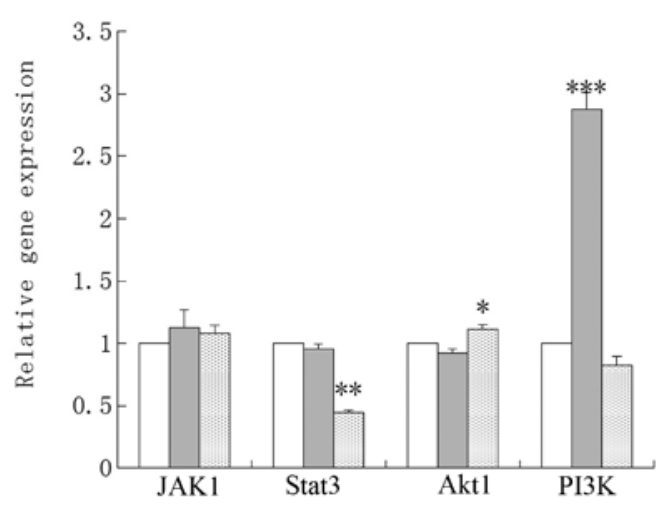

Figure 5. Effects of Aloperine (ALO) on the JAK/Stat3 and PI3K/Akt signaling. (A) The protein levels of JAK1/Stat3 and PI3K/Akt were determined using total protein antibodies by western blotting. (B) Relative expression of JAK1/Stat3 and PI3K/Akt genes examined by qPCR array. Representative up- and downregulated genes are shown. Data are shown as the mean \pm standard deviation (SD), and the relative gene expression was normalized using $\beta$-actin as a control $\left({ }^{*} \mathrm{P}<0.05,{ }^{* *} \mathrm{P}<0.01\right.$ and $\left.{ }^{* * *} \mathrm{P}<0.001\right)$. 
cell cycle progression and inducing apoptosis. Cell cycle arrest at the $\mathrm{G} 2 / \mathrm{M}$ phase correlated with reduced levels of cyclin B1 and D1 protein and increased levels of p53 and p21, while the apoptotic effects of ALO were associated with the upregulation of Bax and a decrease in Bcl-2 protein levels. Moreover, ALO-induced apoptosis may be involved in the inhibition of JAK/Stat 3 and PI3K/Akt signaling.

Apoptosis and cell cycle arrest are two important events involved in anticancer drug treatment. Apoptosis is the result of a highly complex series of events involving cell chromatin condensation, DNA fragmentation, and cell shrinkage (20). Based on this study, apoptosis may be involved in the inhibition of cell proliferation by ALO. ALO inhibited cell proliferation, leading to morphological changes, enhanced loss of plasma membrane polarity, and long-term clonogenic inhibition.

Cells undergo apoptosis through the extrinsic pathway (death receptor pathway) or intrinsic pathway (the mitochondrial pathway) (21). Mitochondria play a crucial role in apoptotic regulation and the mitochondrial dysfunction that occurs during apoptosis $(22,23)$. The Bcl-2 family of proteins promotes cell death by modulating the mitochondrial release of proapoptotic factors and acts as a critical life-death decision point within the common pathway of apoptosis $(24,25)$. In this study, we observed the downregulation of proapoptotic Bax and upregulation of antiapoptotic Bcl-2 proteins in ALO-treated HCT116 cells (Fig. 4C and D).

Cell growth is defined by several genetically defined checkpoints to ensure its coordinated progression through the different stages of the cell cycle and to monitor DNA integrity (26). Cell-cycle arrest in response to stress is integral to the maintenance of genomic integrity. The control mechanisms that restrain cell-cycle transition or induce apoptotic signaling pathways after cell stress are known as cell-cycle checkpoints (27). The analysis of the cell cycle with different concentrations of ALO in HCT116 cells revealed a higher number of cells in the G2/M and $\mathrm{S}$ phase, whereas the number of cells in the G0/G1 phase decreased compared with the untreated cells (Fig. 3B). These results indicate that ALO inhibits cell proliferation via $\mathrm{G} 2 / \mathrm{M}$ phase arrest in a dose-dependent manner. The G2 checkpoint is regulated by the activation of multiple pathways that act together to inhibit the activity of the cyclin B1/cdc2 kinase complex. p53 plays an important role in the regulation of the G2 checkpoint (27). D-type cyclins play an important role in tumor development and are overexpressed in various types of cancer, including breast, colon, and thyroid cancer, as well as melanoma (28). p53 and p21 appear to be essential for maintaining the G2 checkpoint in human cells (29). The accumulation of $\mathrm{G} 2 / \mathrm{M}$ phase cells resulting from ALO treatment led us to determine the expression level of cell cycle regulators. ALO induced cell cycle retardation in the $\mathrm{G} 2 / \mathrm{M}$ phase by increasing the expression of p21 and p53 and by suppressing cyclin B1 and D1 (Fig. 3C). Therefore, our results suggest that apoptosis induction and G2 phase cell cycle arrest both contributed to the anticancer activity of ALO.

Stat proteins are involved in the regulation of fundamental cell processes, such as cell growth, differentiation, and survival (30). Stat 3 is often activated in many types of human cancer, including colon cancer. The inhibition of cancer cell growth by blocking the signaling to Stat 3 demonstrated that
Stat 3 is crucial to the survival and growth of tumor cells and is an attractive therapeutic target for cancer (31-33). Unphosphorylated Stat 3 activates the transcription of Stat3 target genes and plays an important role in oncogenesis (34). The inhibition of the JAK/Stat 3 signaling pathway may be an important mechanism for apoptosis (18). As anticipated, in the present study, ALO downregulated Stat 3 protein and mRNA levels in HCT116 cells (Fig. 5).

The PI3K/Akt signaling pathway is an important regulator of proliferation, differentiation, and metastasis and plays a significant role in apoptosis $(35,36)$. Additionally, the PI3K/Akt signaling pathway is a potential therapeutic target of cancer (37). To elucidate the mechanism involved in ALO-induced cell death, the effects of ALO on PI3K/Akt were examined. PI3KC3 protein was largely affected.

Our experiments mainly aimed at studying the anticancer effect of ALO and the mechanisms of apoptosis and the cell cycle. Other pathways involved in the anticancer properties of the alkaloid compounds, including those that mediate DNA damage, and the relationship between the PI3K/Akt and JAK-Stat3 signaling pathways, remain to be investigated.

In conclusion, compared with the other tested alkaloids, ALO had the most potent effects against HCT116 colon cancer cells. The pure ALO compound exerts an anti-proliferative effect by inducing G2/M cell cycle arrest and apoptosis as well as activating the mitochondrial death pathway in HCT116 cells. In addition, ALO-induced apoptosis may be involved in the inhibition of the JAK/Stat 3 and PI3K/Akt signaling pathways. These findings suggest that ALO should be studied further as an agent of chemotherapeutic activity in human colon cancer.

\section{Acknowledgements}

This study was partly supported by grants from the Natural Science Foundation of China (no. 81001701 to Dr L.L.); the Project of Zhujiang New Star of Guangzhou Science and Technology Project (no. 2013J2200030 to Dr L.L.).

\section{References}

1. Siegel R, Naishadham D and Jemal A: Cancer statistics, 2013. CA Cancer J Clin 63: 11-30, 2013.

2. Ali R, Mirza Z, Ashraf GM, et al: New anticancer agents: recent developments in tumor therapy. Anticancer Res 32: 2999-3005, 2012.

3. Parkinson DR, Arbuck SG, Moore T, Pluda JM and Christian MC: Clinical development of anticancer agents from natural products. Stem Cells 12: 30-43, 1994.

4. Tsukagoshi S: Recent trend on the development of new anticancer drugs. Gan To Kagaku Ryoho 20: 425-431, 1993 (In Japanese).

5. Wang H, Guo S, Qian D, Qian Y and Duan JA: Comparative analysis of quinolizidine alkaloids from different parts of Sophora alopecuroides seeds by UPLC-MS/MS. J Pharm Biomed Anal 67-68: 16-21, 2012.

6. Zhang L, Jiang $\mathrm{J}$ and Tan R: Effects of matrine on telomerase activity and cell cycle in K562 cell. Zhonghua Zhong Liu Za Zhi 20: 328-329, 1998 (In Chinese)

7. Zhang LP, Jiang JK, Tam JW, et al: Effects of matrine on proliferation and differentiation in K-562 cells. Leuk Res 25: 793-800, 2001.

8. Zhang Z, Wang X, Wu W, et al: Effects of matrine on proliferation and apoptosis in gallbladder carcinoma cells (GBC-SD). Phytother Res 26: 932-937, 2012.

9. Yuan C,Lu SQ and Yao X: Effects of oxymatrine on the antitumor activity and toxicity of cyclophosphamide in mice. Yao Xue Xue Bao 22: 245-249, 1987. 
10. Li XM, Wu YG, Chen SL, Pan DX, Wu JN and Yu YH: Antitumor action of sophoridine. Zhongguo Yao Li Xue Bao 8: 153-158, 1987 (In Chinese).

11. Liang L, Wang XY, Zhang XH, et al: Sophoridine exerts an anticolorectal carcinoma effect through apoptosis induction in vitro and in vivo. Life Sci 91: 1295-1303, 2012.

12. Yuan XY, Liu W, Zhang P, Wang RY and Guo JY: Effects and mechanisms of aloperine on 2, 4-dinitrofluorobenzene-induced allergic contact dermatitis in BALB/c mice. Eur J Pharmacol 629: 147-152, 2010.

13. Lin Z, Huang CF, Liu XS and Jiang J: In vitro anti-tumour activities of quinolizidine alkaloids derived from Sophora flavescens Ait. Basic Clin Pharmacol Toxicol 108: 304-309, 2011.

14. Zhou CC, Gao HB, Sun XB, et al: Anti-inflammatory and anti-allergic action of aloperine. Zhongguo Yao Li Xue Bao 10: 360-365, 1989 (In Chinese).

15. Lin WC and Lin JY: Five bitter compounds display different antiinflammatory effects through modulating cytokine secretion using mouse primary splenocytes in vitro. J Agric Food Chem 59: 184-192, 2011

16. Franken NA, Rodermond HM, Stap J, Haveman J and van Bree C: Clonogenic assay of cells in vitro. Nat Protoc 1: 2315-2319, 2006

17. Xi S, Gooding WE and Grandis JR: In vivo antitumor efficacy of STAT3 blockade using a transcription factor decoy approach implications for cancer therapy. Oncogene 24: 970-979, 2005.

18. Zhu Z, Li E, Liu Y, et al: Inhibition of Jak-STAT3 pathway enhances bufalin-induced apoptosis in colon cancer SW620 cells. World J Surg Oncol 10: 228, 2012.

19. Lu Y, Zhou J, Xu C, et al: JAK/STAT and PI3K/AKT pathways form a mutual transactivation loop and afford resistance to oxidative stress-induced apoptosis in cardiomyocytes. Cell Physiol Biochem 21: 305-314, 2008.

20. Debatin KM and Krammer PH: Death receptors in chemotherapy and cancer. Oncogene 23: 2950-2966, 2004.

21. Jin Z and El-Deiry WS: Overview of cell death signaling pathways. Cancer Biol Ther 4: 139-163, 2005.

22. Hu W, Shen T and Wang MH: Cell cycle arrest and apoptosis induced by methyl 3,5-dicaffeoyl quinate in human colon cancer cells: Involvement of the PI3K/Akt and MAP kinase pathways. Chem Biol Interact 194: 48-57, 2011.

23. Itoh K, Hase H, Kojima H, Saotome K, Nishioka K and Kobata T: Central role of mitochondria and p53 in Fas-mediated apoptosis of rheumatoid synovial fibroblasts. Rheumatology (Oxford) 43 : $277-285,2004$
24. Reed JC: Bcl-2 family proteins. Oncogene 17: 3225-3236, 1998.

25. Tsujimoto Y: Role of Bcl-2 family proteins in apoptosis: apoptosomes or mitochondria? Genes Cells 3: 697-707, 1998.

26. Brooks $\mathrm{G}$ and La Thangue NB: The cell cycle and drug discovery: the promise and the hope. Drug Discov Today 4: 455-464, 1999

27. Flatt PM and Pietenpol JA: Mechanisms of cell-cycle checkpoints: at the crossroads of carcinogenesis and drug discovery. Drug Metab Rev 32: 283-305, 2000.

28. Kwak YT, Radaideh SM, Ding L, et al: Cells lacking IKK $\alpha$ show nuclear cyclin D1 overexpression and a neoplastic phenotype: role of IKK $\alpha$ as a tumor suppressor. Mol Cancer Res 9: 341-349, 2011.

29. Bunz F, Dutriaux A, Lengauer C, et al: Requirement for p53 and p21 to sustain G2 arrest after DNA damage. Science 282: 1497-1501, 1998

30. Darnell JE Jr, Kerr IM and Stark GR: Jak-STAT pathways and transcriptional activation in response to IFNs and other extracellular signaling proteins. Science 264: 1415-1421, 1994.

31. Turkson J: STAT proteins as novel targets for cancer drug discovery. Expert Opin Ther Targets 8: 409-422, 2004.

32. Lin L, Liu A, Peng Z, et al: STAT3 is necessary for proliferation and survival in colon cancer-initiating cells. Cancer Res 71: 7226-7237, 2011.

33. Turkson J and Jove R: STAT proteins: novel molecular targets for cancer drug discovery. Oncogene 19: 6613-6626, 2000.

34. Nishimoto A, Kugimiya N, Hosoyama T, Enoki T, Li TS and Hamano K: JAB1 regulates unphosphorylated STAT3 DNA-binding activity through protein-protein interaction in human colon cancer cells. Biochem Biophys Res Commun 438: $513-518,2013$.

35. Jia SS, Xi GP, Zhang M, et al: Induction of apoptosis by D-limonene is mediated by inactivation of Akt in LS174T human colon cancer cells. Oncol Rep 29: 349-354, 2013.

36. Harashima N, Inao T, Imamura R, Okano S, Suda T and Harada M: Roles of the PI3K/Akt pathway and autophagy in TLR3 signaling-induced apoptosis and growth arrest of human prostate cancer cells. Cancer Immunol Immunother 61: 667-676, 2012.

37. Liu Y, Mei C, Sun L, et al: The PI3K-Akt pathway regulates calpain 6 expression, proliferation, and apoptosis. Cell Signal 23: 827-836, 2011. 